\title{
ORBIFOLD HODGE NUMBERS OF THE WREATH PRODUCT ORBIFOLDS
}

\author{
WEIQIANG WANG AND JIAN ZHOU
}

\begin{abstract}
We prove that the wreath product orbifolds studied earlier by the first author provide a large class of higher dimensional examples of orbifolds whose orbifold Hodge numbers coincide with the ordinary ones of suitable resolutions of singularities. We also make explicit conjectures on elliptic genera for the wreath product orbifolds.
\end{abstract}

Key words: Wreath products, (orbifold) Hodge numbers, Hilbert schemes, elliptic genera.

2000 Mathematics Subject Classifications: 14J, 58J.

\section{INTRODUCTION}

In the study of orbifold string theory, Dixon, Harvey, Vafa and Witten 12 introduced the notion of orbifold Euler number for a smooth manifold $Y$ acted on by a finite group $G$ and raised the question on the existence of a resolution of singularities of $M / G$ whose ordinary Euler number coincides with the orbifold Euler number. The orbifold Euler number has subsequently been interpreted as the Euler number for equivariant $K$-theory, cf. Atiyah-Segal [1]. The notion of orbifold Euler number has been further refined to give rise to the notion of orbifold Hodge numbers [26, 29] and more generally the stringy Hodge numbers [5]. The orbifold Hodge numbers of an orbifold are then conjectured to coincide with the ordinary Hodge numbers of a suitable resolution of the orbifold. For recent related

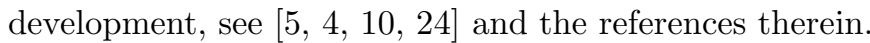

A well-known series of examples with such a property is provided by the symmetric product of a surface which admits a resolution of singularities given by the Hilbert scheme of points. In this case the orbifold Euler number calculated by Hirzebruch and Höfer 19 matches with the Euler number of the Hilbert scheme found earlier by Göttsche [15]. It is further shown by Göttsche [16] that the orbifold Hodge numbers matches with the Hodge numbers of the Hilbert scheme calculated by Göttsche and Soergel [17] (also see Cheah [8]). The same method has been used by the second author [30], also for the calculation for higher dimensional complex manifolds. . $^{2}$

The wreath product orbifolds, which are generalizations of the symmetric products, were shown by the first author [27] (also see [14, 28]) to have deep connections with Hilbert schemes of surfaces and vertex representations of infinite dimensional

\footnotetext{
${ }^{1}$ Seeing the math review (99c:14022) of 16 ] but not the paper itself when he was writing 30 , the second author got the wrong impression that Göttsche proved his result by establishing the strong McKay correspondence for symmetric products. This misunderstanding has been clarified when we actually looked into the paper 16] during the preparation of the present paper.
} 
Lie algebras. More explicitly, if $Y$ is a smooth manifold acted upon by a finite group $G$, then there exists a natural action on the $n$-th Cartesian product $Y^{n}$ by the wreath product $G_{n}$ (which is the semidirect product of the symmetric group $S_{n}$ and the product group $\left.G^{n}\right)$. The orbifold Euler number for $Y^{n} / G_{n}$ has been explicitly calculated in loc. cit.. If in addition we assume that $Y$ is a quasi-projective surface and $X$ is a resolution of singularities of the orbifold $Y / G$, then the following commutative diagram

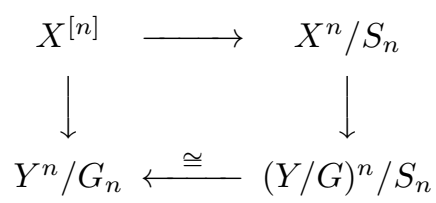

implies that the Hilbert scheme $X^{[n]}$ is a resolution of singularities of the orbifold $X^{n} / G_{n}$. It has been shown 27 that if the ordinary Euler number of $X$ equals the orbifold Euler number of $Y / G$ then the ordinary Euler number of $X^{[n]}$ equals the orbifold Euler number of $X^{n} / G_{n}$ for all $n$. When $G$ is the trivial group and $X$ equals $Y$, one recovers the case of symmetric products.

The purpose of the present paper is to point out that the wreath product orbifolds also provide a large class of new higher dimensional examples which verify the orbifold Hodge number conjecture. More precisely, we show that if $Y$ is a quasi-projective surface and $X$ is a resolution of singularities of $Y / G$ such that the ordinary Hodge numbers of $X$ coincide with the orbifold Hodge numbers of the orbifold $Y / G$, then the orbifold Hodge numbers of the orbifold $Y^{n} / G_{n}$ coincide with the Hodge numbers of the Hilbert scheme $X^{[n]}$, which is a resolution of singularities. Our proof rely on the analysis of fixed-point set structures of the wreath product action on $Y^{n}$ (cf. 27]). As in [30] our calculation of the orbifold Hodge numbers for $Y^{n} / G_{n}$ actually works for any complex $G$-manifold of even dimension.

In a very recent paper [7], Bryan, Donagi and Leung pointed out a series of examples (besides the well-known symmetric products) verifying the orbifold Hodge numbers conjecture. It turns out that their examples correspond to our special case when $Y$ is an abelian surface, $G$ is $\mathbb{Z}_{2}$, and $X$ is the Kummer $K 3$ surface. They remarked that most examples in literature are lower dimensional and no other higher dimensional examples known to them. Their remarks are largely responsible for us to decide to write up the results on the wreath product orbifolds which have been known to us for some time. We do not know any other higher dimensional examples which verify the orbifold Hodge number conjecture.

To conclude we also make two explicit conjectures on elliptic genera for wreath product orbifolds. These are motivated and in turn generalize the work of Dijkgraaf, Moore, Verlinde and Verlinde [11] on elliptic genera for symmetric products.

The layout of this paper is as follows. In section 2, we recall the fixed-point set structures of the wreath product action, and the definition of the orbifold Hodge numbers, for both the compact and noncompact situations. In section 3, we prove our main results, Theorem 3.1 on the orbifold Hodge numbers of wreath product orbifolds and Theorem 3.2 on the verification of orbifold Hodge number conjecture. In section 4 , we provide various examples illustrating our main results and in addition formulate two conjectures on elliptic genera. 


\section{PRELIMINARIES ON THE WREATH PRODUCT AND ORBIFOLD HODGE NUMBERS}

In this section, we first review the definition of a wreath product $G_{n}$ associated to a finite group $G$, and the descriptions of conjugacy classes and centralizers for $G_{n}$, cf. e.g. 20, 23. We also describe fixed-point sets for the action of $G_{n}$ on the $n$-th Cartesian product of a $G$-manifold, following [27]. We then recall the definition of orbifold Hodge numbers, cf. [29, 5.

2.1. The wreath product action on $Y^{n}$. Let $G$ be a finite group and denote by $G_{*}$ the set of conjugacy classes of $G$. Let $G^{n}=G \times \ldots \times G$ be the direct product of $n$ copies of $G$. Denote by $[g]$ the conjugacy class of $g \in G$. The symmetric group $S_{n}$ acts on $G^{n}$ by permuting the $n$ factors: $s\left(g_{1}, \ldots, g_{n}\right)=\left(g_{s^{-1}(1)}, \ldots, g_{s^{-1}(n)}\right)$. The wreath product $G_{n}=G \imath S_{n}$ is defined to be the semidirect product $G^{n} \rtimes S_{n}$ of $G^{n}$ and $S_{n}$, namely the multiplication on $G_{n}$ is given by $(g, s)(h, t)=(g . s(h), s t)$, where $g, h \in G^{n}, s, t \in S_{n}$. Note when $G$ is the trivial one-element group the wreath product $G_{n}$ reduces to $S_{n}$, and when $G$ is $\mathbb{Z}_{2}$ the wreath product $G_{n}$ is the hyperoctahedral group, the Weyl group of type $C$.

Given $a=(g, s) \in G_{n}$ where $g=\left(g_{1}, \ldots, g_{n}\right)$, we write $s \in S_{n}$ as a product of disjoint cycles: if $z=\left(i_{1}, \ldots, i_{r}\right)$ is one of them, the cycle-product $g_{i_{r}} g_{i_{r-1}} \ldots g_{i_{1}}$ of $a$ corresponding to the cycle $z$ is determined by $g$ and $z$ up to conjugacy. For each $c \in G_{*}$ and each integer $r \geq 1$, let $m_{r}(c)$ be the number of $r$-cycles in $s$ whose cycle-product lies in $c$. Denote by $\rho(c)$ the partition having $m_{r}(c)$ parts equal to $r$ $(r \geq 1)$ and denote by $\rho=(\rho(c))_{c \in G_{*}}$ the corresponding partition-valued function on $G_{*}$. Note that $\|\rho\|:=\sum_{c \in G_{*}}|\rho(c)|=\sum_{c \in G_{*}, r \geq 1} r m_{r}(c)=n$, where $|\rho(c)|$ is the size of the partition $\rho(c)$. Thus we have defined a map from $G_{n}$ to $\mathcal{P}_{n}\left(G_{*}\right)$, the set of partition-valued function $\rho=(\rho(c))_{c \in G_{*}}$ on $G_{*}$ such that $\|\rho\|=n$. The function $\rho$ or the data $\left\{m_{r}(c)\right\}_{r, c}$ is called the type of $a=(g, s) \in G_{n}$. Denote $\mathcal{P}\left(G_{*}\right)=\sum_{n>0} \mathcal{P}_{n}\left(G_{*}\right)$. It is well known (cf. e.g. 20, 23]) that two elements in $G_{n}$ are conjugate to each other if and only if they have the same type.

Let us describe the centralizer $Z_{G_{n}}(a)$ of $a \in G_{n}$, cf. [20, 23, 27]. First we consider the typical case that $a$ has one $n$-cycle. As the centralizers of conjugate elements are conjugate subgroups, we may assume that $a$ is of the form $a=((g, 1, \ldots, 1), \tau)$, where $\tau=(12 \ldots n)$. Denote by $Z_{G}^{\Delta}(g)$, or $Z_{G}^{\Delta_{n}}(g)$ when it is necessary to specify $n$, the following diagonal subgroup of $G^{n}$ (and thus a subgroup of $\left.G_{n}\right)$ :

$$
Z_{G}^{\Delta}(g)=\left\{((h, \ldots, h), 1) \in G^{n} \mid h \in Z_{G}(g)\right\} .
$$

The centralizer $Z_{G_{n}}(a)$ of $a$ in $G_{n}$ is equal to the product $Z_{G}^{\Delta}(g) \cdot\langle a\rangle$, where $\langle a\rangle$ is the cyclic subgroup of $G_{n}$ generated by $a$.

Take a generic element $a=(g, s) \in G_{n}$ of type $\rho=(\rho(c))_{c \in G_{*}}$, where $\rho(c)$ has $m_{r}(c) r$-cycles $(r \geq 1)$. We may assume (by taking a conjugation if necessary) that the $m_{r}(c) r$-cycles are of the form

$$
g_{u r}(c)=\left((g, 1, \ldots, 1),\left(i_{u 1}, \ldots, i_{u r}\right)\right), \quad 1 \leq u \leq m_{r}(c), g \in c .
$$

Denote $g_{r}(c)=((g, 1, \ldots, 1),(12 \ldots r))$. Throughout the paper, $\prod_{c, r}$ is understood as the product $\prod_{c \in G_{*}, r \geq 1}$. The centralizer $Z_{G_{n}}(a)$ of $a \in G_{n}$ is isomorphic to a direct product of the wreath products

$$
\prod_{c, r}\left(Z_{G_{r}}\left(g_{r}(c)\right) 2 S_{m_{r}(c)}\right) .
$$


Furthermore $Z_{G_{r}}\left(g_{r}(c)\right)$ is isomorphic to $Z_{G}^{\Delta_{r}}(g) \cdot\left\langle g_{r}(c)\right\rangle$.

For a $G$-space $Y$, we define an action of $G_{n}$ on $Y^{n}$ as follows. Given $a=$ $\left(\left(g_{1}, \ldots, g_{n}\right), s\right)$, we let

$$
a .\left(x_{1}, \ldots, x_{n}\right)=\left(g_{1} x_{s^{-1}(1)}, \ldots, g_{n} x_{s^{-1}(n)}\right)
$$

where $x_{1}, \ldots, x_{n} \in Y$.

Next we recall the description of the fixed point set $\left(Y^{n}\right)^{a}$ for $a \in G_{n}$, cf. 27]. Let us first look at the typical case $a=((g, 1, \ldots, 1), \tau) \in G_{n}$. Note that the centralizer group $Z_{G}(g)$ preserves the $g$-fixed point set $X^{g}$. The fixed point set is

$$
\left(Y^{n}\right)^{a}=\left\{(x, \ldots, x) \in Y^{n} \mid x=g x\right\}
$$

which can be naturally identified with $Y^{g}$. The action of $Z_{G_{n}}(a)$ on $\left(Y^{n}\right)^{a}$ can be identified canonically with that of $Z_{G}(g)$ on $Y^{g}$ together with the trivial action of the cyclic group $\langle a\rangle$. Thus $\left(X^{n}\right)^{a} / Z_{G_{n}}(a)$ can be identified with $X^{g} / Z_{G}(g)$.

All $Z_{G}(g)$ are conjugate and all $X^{g}$ are homeomorphic to each other for different representatives $g$ in a fixed conjugacy class $c \in G_{*}$. Also the orbit space $X^{g} / Z_{G}(g)$ can be identified with each other by conjugation for different representatives of $g$ in $c \in G_{*}$. We agree to denote $Z_{G}(g)$ (resp. $\left.Y^{g}, Y^{g} / Z_{G}(g)\right)$ by $Z_{G}(c)$ (resp. $Y^{c}$, $Y^{c} / Z_{G}(c)$ ) by abuse of notations. Similar remarks apply to other situations below when the choice of representatives in a conjugacy class is irrelevant.

For an element $a \in G_{n}$ of type $\left\{m_{r}(c)\right\}$, the fixed-point set $\left(Y^{n}\right)^{a}$ can be naturally identified with $\prod_{c, r}\left(Y^{c}\right)^{m_{r}(c)}$. Furthermore the orbit space $\left(Y^{n}\right)^{a} / Z_{G_{n}}(a)$ can be naturally identified with

$$
\prod_{c, r} S^{m_{r}(c)}\left(Y^{c} / Z_{G}(c)\right)
$$

where $S^{m}(X)$ denotes the $m$-th symmetric product $X^{m} / S_{m}$.

2.2. Definition of orbifold Hodge numbers. Let $Y$ be a compact complex manifold of complex dimension $d$ acted on by a finite group $G$ of automorphisms. For each conjugacy class $c=[g] \in G_{*}$, let $Y_{1}^{g}, \cdots, Y_{N_{c}}^{g}$ be the connected components of the fixed-point set $Y^{g}$. Zaslow 29] defined a shift number $F_{\alpha}^{g}$ associated to each component $Y_{\alpha}^{g}$ as follows. On the tangent space to each point in $Y_{\alpha}^{g}, g$ acts as a diagonal matrix $\operatorname{diag}\left(e^{2 \pi \sqrt{-1} \theta_{1}}, \cdots, e^{2 \pi \sqrt{-1} \theta_{d}}\right)$, where $0 \leq \theta_{i}<1$. Then

$$
F_{\alpha}^{g}=\sum_{j=1}^{d} \theta_{j}
$$

In general, $F_{\alpha}^{g}$ is just a rational number. However, there are many occasions when it is an integer, e.g., when $g$ acts on the tangent space by a matrix in $S L(n, \mathbb{C})$.

Remark 2.1. In the case when $Y$ is a complex surface, the shift $F_{\alpha}^{g}$ is an integer only if the component $Y_{\alpha}^{g}$ is either an isolated point or two dimensional. Indeed a finite subgroup $G$ of $G L(2, \mathbb{C})$ acting on $\mathbb{C}^{2}$ has integer shifts if and only if $G$ lies in $S L(2, \mathbb{C})$. That is, the shift $F_{\alpha}^{g}=\theta_{1}+\theta_{2}$ is an integer if and only if $\operatorname{det} g=e^{2 \pi \sqrt{-1}\left(\theta_{1}+\theta_{2}\right)}=1$.

In the case all the shifts are integers, the orbifold Hodge numbers of the orbifold $Y / G$ are defined to be

$$
h^{s, t}(Y, G)=\sum_{c \in G_{*}} \sum_{\alpha_{c}=1}^{N_{c}} h^{s-F_{\alpha_{c}}^{c}, t-F_{\alpha_{c}}^{c}}\left(Y_{\alpha_{c}}^{c} / Z(c)\right) .
$$


The ordinary Dolbeault cohomology for an orbifold is given by (cf. Satake 25])

$$
H^{*, *}(Y / G) \cong H^{*, *}(Y)^{G} .
$$

Clearly the orbifold Hodge numbers can now be regarded as the dimensions of the corresponding orbifold cohomology groups (cf. [29, 30])

$$
H^{*, *}(Y, G)=\sum_{c \in G_{*}} \bigoplus_{\alpha_{c}=1}^{N_{c}} H^{*, *}\left(Y_{\alpha_{c}}^{c} / Z_{c}\right)\left\{F_{\alpha_{c}}^{c}\right\} .
$$

Here and below we adopt the convention that if $V=\oplus_{s, t \in \mathbb{Z}} V^{s, t}$ is a bigraded vector space, then $V\{n\}$ is the bigraded vector space with $(V\{n\})^{s, t}=V^{s-n, t-n}$.

It is convenient to form the generating function of bigraded spaces

$$
H(Y, G ; x, y)=\sum_{s, t} H^{s, t}(Y, G) x^{s} y^{t},
$$

whose graded dimension is given by the orbifold Hodge polynomial

$$
h(Y, G ; x, y)=\sum_{s, t} h^{s, t}(Y, G) x^{s} y^{t} .
$$

Then we can rewrite the definition of orbifold cohomology groups as

$$
\begin{aligned}
H(Y, G ; x, y) & =\sum_{c \in G_{*}} \bigoplus_{\alpha_{c}=1}^{N_{c}} H\left(Y_{\alpha_{c}}^{c} / Z_{c} ; x, y\right)\left\{F_{\alpha_{c}}^{c}\right\} \\
& =\sum_{c \in G_{*}} \bigoplus_{\alpha_{c}=1}^{N_{c}} H\left(Y_{\alpha_{c}}^{c} / Z_{c} ; x, y\right)(x y)^{F_{\alpha_{c}}^{c}} .
\end{aligned}
$$

For later use we define the orbifold virtual Hodge polynomial

$$
e(Y, G ; x, y)=\sum_{s, t}(-1)^{s+t} h^{s, t}(Y, G) x^{s} y^{t} .
$$

We also define the usual virtual Hodge polynomial for the Hodge numbers $h^{s, t}(Y)$ associated to smooth $Y$ by letting $e(Y ; x, y)=\sum_{s, t}(-1)^{s+t} h^{s, t}(Y) x^{s} y^{t}$.

2.3. The definition of orbifold virtual Hodge numbers. We now indicate how to extend the above definitions to the case of smooth quasi-projective varieties by using Deligne's theory of mixed Hodge structures [9]. Recall that a (pure) Hodge structure of weight $m$ on a complex vector space $H$ with a real structure is a direct sum decomposition:

$$
H=\bigoplus_{s+t=m} H^{s, t}
$$

such that $\bar{H}^{s, t}=H^{t, s}$ for all pairs $(s, t)$. A mixed Hodge structure (MHS) on $H$ consists of two filtrations

$$
0 \subset \cdots \subset W_{m-1} \subset W_{m} \subset W_{m+1} \subset \cdots \subset H
$$

the 'weight filtration', and

$$
H \supset \cdots \supset F^{p-1} \supset F^{p} \supset F^{p+1} \supset \cdots \supset 0,
$$


the 'Hodge filtration', such that the filtration induced by the latter on $G r_{m}\left(W_{*}\right)=$ $W_{m} / W_{m-1}$ defines a Hodge structure of weight $m$, for each $m$.

Define

$$
I^{s, t}=F^{s} \cap W_{s+t} \cap\left[\overline{F^{t}} \cap W_{s+t}+\sum_{i \geq 2} \overline{F^{t-i+1}} \cap W_{s+t-i}\right] ;
$$

Then $I^{s, t} \subset W_{s+t}$ maps isomorphically to the $(s, t)$ component in $G r_{s+t}\left(W_{*}\right)$. One can show that

$$
F^{s}(H)=\bigoplus_{s^{\prime} \geq s} \bigoplus_{t} I^{s^{\prime}, t}(H), \quad W_{m}(H)=\bigoplus_{s+t \leq m} I^{s, t}(H) .
$$

It can be shown that $\left\{I^{s, t}\right\}$ is a splitting of $H$ characterized by the property that

$$
I^{s, t} \cong \overline{I^{t, s}}\left(\bmod \bigoplus_{s^{\prime}<s, t^{\prime}<t} I^{s^{\prime}, t^{\prime}}\right)
$$

(cf. 90). We will refer to this splitting as the canonical splitting. Define

$$
h^{s, t}(H)=\operatorname{dim} I^{s, t}(H) .
$$

Let $V=\oplus_{k \geq 0} V^{k}$ be a graded vector space, with $\operatorname{dim} V^{k}<\infty$ for all $k$. Assume that each $V^{k}$ is endowed with a MHS. We will refer to such a space as a graded vector space with MHS. The virtual Hodge numbers and the virtual Hodge polynomial of $V$ are defined by

$$
\begin{aligned}
& e^{s, t}(V)=\sum_{k \geq 0}(-1)^{k} h^{s, t}\left(V^{k}\right), \\
& e_{x, y}(V)=\sum_{s, t} e^{s, t}(V) x^{s} y^{t} .
\end{aligned}
$$

Alternatively, we have the splitting:

$$
V=\bigoplus_{k \geq 0} \bigoplus_{s, t} I^{s, t}\left(V^{k}\right)
$$

Consider the generating function

$$
e_{x, y, z}(V)=\sum_{k \geq 0} \sum_{s, t} \operatorname{dim} I^{s, t}\left(V^{k}\right) x^{s} y^{t} z^{k}
$$

Then $e_{x, y}(V)=e_{x, y,-1}(V)$. We will use the following convention: for a graded vector space with MHS $V=\oplus_{k \geq 0} V^{k}$ and a positive integer $n, V\{n\}$ is the graded vector space with MHS such that for each $k$,

$$
W_{m}\left(V^{k}\{n\}\right)=W_{m-2 n}\left(V^{k}\{n\}\right), \quad F^{p}\left(V^{k}\{n\}\right)=F^{p-n}\left(V^{k}\{n\}\right) .
$$

It is straightforward to see that $e^{s, t}(V\{n\})=e^{s-n, t-n}(V)$, and so

$$
e_{x, y}(V\{n\})=(x y)^{n} e_{x, y}(V) .
$$

Deligne [9] has shown that for an arbitrary complex algebraic variety $Y$, the cohomology $H^{k}(Y)$ carries a MHS which coincides with the classical pure Hodge structure in the case of smooth projective varieties. Hence one can define the virtual Hodge number of $Y$

$$
e^{s, t}(Y)=e^{s, t}\left(H^{*}(Y)\right)
$$


and the virtual Hodge polynomial of $Y$

$$
e(Y ; x, y)=e_{x, y}\left(H^{*}(Y)\right) .
$$

Assume that $(Y, G)$ is a pair consisting of a smooth quasi-projective variety $Y$ and a finite subgroup $G$ of automorphisms of $Y$. Then by functorial property, there is an induced action of $G$ on the MHS on $H^{*}(Y)$ by automorphisms. By taking the invariant parts, we obtain a MHS on each $H^{k}(Y / G)$. One can also achieve this by taking a smooth compactification $\bar{Y}$ such that $D=\bar{Y}-Y$ is a divisor with normal crossing singularities and such that the $G$-action extends to $\bar{Y}$. Then the MHS on $H^{*}(Y / G)$ is obtained by using $\left(\Omega_{Y}^{*}\langle D\rangle\right)^{G}$, the invariant part of the complex of differential forms with logarithmic poles. Using the above MHS on $H^{*}(Y / G)$, we can now define $e^{p, q}(Y / G)$. Similar to the closed case (cf. (3)), we define the orbifold virtual Hodge number as follows:

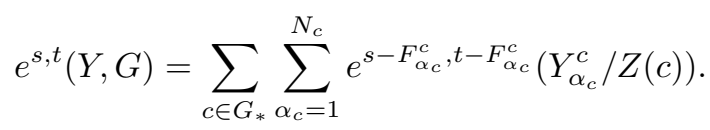

We also define the orbifold virtual Hodge polynomial:

$$
e(Y, G ; x, y)=\sum_{s, t} e^{s, t}(Y, G) x^{s} y^{t} .
$$

It is clear that $e(Y, G ; x, y)$ is the virtual Hodge polynomial of

$$
H^{*}(Y, G)=\sum_{c \in G_{*}} \bigoplus_{\alpha_{c}=1}^{N} H^{*}\left(Y_{\alpha_{c}}^{c} / Z(c)\right)\left\{F_{\alpha_{c}}^{c}\right\}
$$

(cf (5)), where both sides are understood as graded vector spaces with MHS.

Remark 2.2. One can replace $H^{*}(Y)$ by the cohomology with compact support $H_{c}^{*}(Y)$ in the above definitions.

\section{The ORBIFOld Hodge Numbers of WreAth PRODUCT ORBIFOldS}

In this section, we calculate explicitly the ordinary and orbifold Hodge numbers of wreath product orbifolds $Y^{n} / G_{n}$ associated to an even-dimensional orbifold $Y / G$.

3.1. Two simple lemmas. Let $V=\oplus_{s, t \in \mathbb{Z}_{+}} V^{s, t}$ be a bigraded complex vector space, such that $\operatorname{dim} V^{s, t}<\infty$ for all $s, t$, where $\mathbb{Z}_{+}$is the set of non-negative integers. We introduce the generating function

$$
h_{x, y}(V)=\sum_{s, t \in \mathbb{Z}_{+}}\left(\operatorname{dim} V^{s, t}\right) x^{s} y^{t} .
$$

For example, when $V$ is the total Dolbeault cohomology group $H(Y)$, then $h_{x, y}(V)$ is its associated Hodge polynomial $h(Y ; x, y)$. When $V$ is the total orbifold Dolbeault cohomology group $H(Y, G)$, then $h_{x, y}(V)$ is its associated orbifold Hodge polynomial $h(Y, G ; x, y)$. It is actually more convenient to work with $e_{x, y}(V)=$ $h_{-x,-y}(V)$. It is easy to see that

$$
\begin{aligned}
& e_{x, y}\left(V_{1} \oplus V_{2}\right)=e_{x, y}\left(V_{1}\right)+e_{x, y}\left(V_{2}\right), \\
& e_{x, y}\left(V_{1} \otimes V_{2}\right)=e_{x, y}\left(V_{1}\right) e_{x, y}\left(V_{2}\right) .
\end{aligned}
$$

The graded symmetric algebra of $V$ is by definition

$$
S(V)=T(V) / I
$$


where $T(V)$ is the tensor algebra of $V, I$ is the ideal generated by elements of the form

$$
v \otimes w-(-1)^{(s+t)(p+q)} w \otimes v, \quad v \in V^{s, t}, w \in V^{p, q} .
$$

The bigrading on $V$ induces a bigrading on $T(V)$ and also on $S(V)$, and hence $e_{x, y}(S(V))$ makes sense. Note that for bigraded vector spaces $V_{1}$ and $V_{2}$, we have $S\left(V_{1} \oplus V_{2}\right) \cong S\left(V_{1}\right) \otimes S\left(V_{2}\right)$. Consequently,

$$
e_{x, y}\left(S\left(V_{1} \oplus V_{2}\right)\right)=e_{x, y}\left(S\left(V_{1}\right)\right) e_{x, y}\left(S\left(V_{2}\right)\right) .
$$

By introducing a formal variable $q$ to count the degree of symmetric power, we can write formally $S(q V)=\sum_{n \geq 0} S^{n}(V) q^{n}$. By breaking $V$ into one-dimensional subspaces, one can easily prove the following.

Lemma 3.1. For any bigraded vector space $V=\oplus_{s, t \geq 0} V^{s, t}$ with $\operatorname{dim} V^{s, t}<\infty$ for all pairs $(s, t)$, we have

$$
\sum_{n \geq 0} e_{x, y}\left(S^{n}(V)\right) q^{n}=\prod_{s, t} \frac{1}{\left(1-x^{s} y^{t} q\right)^{e^{s, t}(V)}}
$$

where $e^{s, t}(V)=(-1)^{s+t} \operatorname{dim} V^{s, t}$.

For a formal power series $\sum_{r>0} V_{r} q^{r}$, where each $V_{r}$ is a bigraded vector space of weight $r$ such that $\operatorname{dim} V_{r}^{s, t}<\infty$, define

$$
S\left(\sum_{r>0} V_{r} q^{r}\right)=\sum_{m \geq 0} \sum_{\sum_{j=1}^{m} j m_{j}=m} q^{m} \bigotimes_{j=1}^{m} S^{m_{j}}\left(V_{j}\right) .
$$

Formally we have

$$
S\left(\sum_{r>0} V_{r} q^{r}\right)=\bigotimes_{r>0} S\left(V_{r} q^{r}\right)
$$

and

$$
e_{x, y}\left(\sum_{r>0} V_{r} q^{r}\right)=\sum_{r>0} e_{x, y}\left(V_{r}\right) q^{r} .
$$

Then the next lemma follows from Lemma 3.1.

Lemma 3.2. For a sequence $\left\{V_{n}\right\}$ of bigraded vector spaces, we have the following formula:

$$
e_{x, y}\left(S\left(\sum_{n>0} V_{n} q^{n}\right)\right)=\prod_{n>0} \prod_{s, t} \frac{1}{\left(1-x^{s} y^{t} q^{n}\right)^{e^{s, t}\left(V_{n}\right)}}
$$

Remark 3.1. Using the canonical splitting, it is fairly straightforward to generalize Lemma 3.1 and Lemma 3.2 to the case of vector spaces with MHS. 
3.2. The main theorems. Since $G^{n}$ is a normal subgroup of the wreath product $G_{n}=G^{n} \rtimes S_{n}$, it is easy to see by (4) that

$$
H\left(Y^{n} / G_{n} ; x, y\right) \cong H\left(Y^{n} ; x, y\right)^{G^{n} \rtimes S_{n}} \cong S^{n}\left(H(Y ; x, y)^{G}\right) \cong S^{n}(H(Y / G ; x, y)) .
$$

When $Y$ is a compact complex manifold, this is an isomorphism of bigraded vector spaces; when $Y$ is a quasi-projective smooth variety over $\mathbb{C}$, this is an isomorphism of graded vector spaces with MHS. As a consequence of Lemma 3.1 and Remark 3.1. we obtain the following proposition.

Proposition 3.1. If $Y$ is a compact complex manifold or a quasi-projective smooth variety, and $G$ is a finite subgroup of automorphisms on $Y$, then we have the following formula:

$$
\sum_{n \geq 0} e\left(Y^{n} / G_{n} ; x, y\right) q^{n}=\prod_{s, t} \frac{1}{\left(1-x^{s} y^{t} q\right)^{e^{s, t}(Y / G)}} .
$$

The first main result of this paper is the following theorem.

Theorem 3.1. Given a compact complex manifold or a smooth quasi-projective variety $Y$ of even complex dimension d, acted on by a finite group $G$ with integer shifts, we have the following formula for the orbifold Hodge numbers:

$$
\sum_{n=1}^{\infty} e\left(Y^{n}, G_{n} ; x, y\right) q^{n}=\prod_{r=1}^{\infty} \prod_{s, t} \frac{1}{\left(1-x^{s} y^{t} q^{r}(x y)^{(r-1) d / 2}\right)^{e^{s, t}(Y, G)}} .
$$

Proof. We first compute the shifts $F^{c}$ for the orbifold $Y^{n} / G_{n}$ associated to a conjugacy class $c$ in $G_{n}$. Consider the typical class containing

$$
g \imath \sigma_{n}=((g, 1, \cdots, 1),(12 \cdots n))
$$

where $g \in G$. Recall from the previous section that a fixed point in $Y^{n}$ by the action of $g \nmid \sigma_{n}$ is of the form $(x, \ldots, x)$ where $x \in Y^{g}$. Since the calculation can be done locally, we will assume that we take local coordinates $\left(z_{1}, \cdots, z_{d}\right)$ near a point $x \in Y^{g}$ such that the action is given by

$$
g\left(z_{1}, \cdots, z_{d}\right)=\left(e^{2 \pi \sqrt{-1} \theta_{1}} z_{1}, \cdots, e^{2 \pi \sqrt{-1} \theta_{r}} z_{r}, z_{r+1}, \cdots, z_{d}\right)
$$

Equivalently, $g$ is locally given by the diagonal matrix $\operatorname{diag}\left(e^{2 \pi \sqrt{-1} \theta_{1}}, \cdots, e^{2 \pi \sqrt{-1} \theta_{d}}\right)$ where $\theta_{r+1}=\cdots=\theta_{d}=0$. Then on $Y^{n}$ near $(x, \cdots, x), g<\sigma_{n}$ is given by a block diagonal matrix with blocks of the form

$$
\left(\begin{array}{ccccc}
0 & 1 & 0 & \cdots & 0 \\
0 & 0 & 1 & \cdots & 0 \\
\cdots & \cdots & \cdots & \cdots & 0 \\
0 & 0 & 0 & \cdots & 1 \\
e^{2 \pi \sqrt{-1} \theta_{j}} & 0 & \cdots & \cdots & 0
\end{array}\right)
$$

The characteristic polynomial of this matrix is $\lambda^{n}-e^{\sqrt{-1} \theta_{j}}$, hence it has eigenvalues

$$
\lambda_{j k}=e^{2 \pi \sqrt{-1}\left(\theta_{j}+k\right) / n}, \quad k=0, \cdots, n-1 .
$$

Notice that $\lambda_{j k}=1$ if and only $\theta_{j}=k=0$. So the shift for the component of $\left(Y^{n}\right)^{g l \sigma_{n}}$ containing $(x, \ldots, x)$ is given by 


$$
\begin{aligned}
F^{g l \sigma_{n}}(x, \ldots, x) & =\sum_{j=1}^{r} \sum_{k=0}^{n-1} \frac{\theta_{j}+k}{n}+(d-r) \sum_{k=1}^{n-1} \frac{k}{n} \\
& =\sum_{j=1}^{r} \theta_{j}+(n-1) d / 2=F_{\alpha_{c}}^{c}+(n-1) d / 2
\end{aligned}
$$

Here we have assumed that $x \in Y^{g}$ lies in the component $Y_{\alpha_{c}}^{g}\left(\alpha_{c}=1, \ldots, N_{c}\right)$, and $F_{\alpha_{c}}^{c}$ is the shift for the component $Y_{\alpha_{c}}^{c} / Z_{G}(c)$.

For a general conjugacy class containing an element $a$ of type

$$
\rho=\left\{m_{r}(c)\right\}_{r \geq 1, c \in G_{*}}
$$

where $\sum_{r, c} r m_{r}(c)=n$, the description of the fixed-point set $\left(Y^{n}\right)^{a}$ given in (2) implies that the components for $\left(Y^{n}\right)^{a}$ can be listed as

$$
\left(Y^{n}\right)_{\left\{m_{r, c}\left(\alpha_{c}\right)\right\}}^{a}=\prod_{r, c} \prod_{\alpha_{c}=1}^{N_{c}} S^{m_{r, c}\left(\alpha_{c}\right)}\left(Y_{\alpha_{c}}^{c} / Z_{G}(c)\right),
$$

where $\left(m_{r, c}(1), \ldots, m_{r, c}\left(N_{c}\right)\right)$ satisfies $\sum_{\alpha_{c}=1}^{N_{c}} m_{r, c}\left(\alpha_{c}\right)=m_{r}(c)$. Then the shift for the component $\left(Y^{n}\right)_{\left\{m_{r, c}\left(\alpha_{c}\right)\right\}}^{a}$ is given by

$$
F_{\left\{m_{r, c}\left(\alpha_{c}\right)\right\}}=\sum_{r, c} \sum_{\alpha_{c}=1}^{N_{c}} m_{r, c}\left(\alpha_{c}\right)\left(F_{\alpha_{c}}^{c}+(r-1) d / 2\right) .
$$

By using (6), (2), (9) and (7) we have 


$$
\begin{aligned}
& \sum_{n \geq 0} H\left(Y^{n}, G_{n} ; x, y\right) q^{n} \\
= & \sum_{n=0}^{\infty} \bigoplus_{\left\{m_{r}(c)\right\} \in \mathcal{P}_{n}\left(G_{*}\right)} \bigotimes_{r, c} \bigotimes_{\alpha_{c}=1}^{N_{c}} H\left(S^{m_{r, c}\left(\alpha_{c}\right)}\left(Y_{\alpha_{c}}^{c} / Z_{G}(c)\right) ; x, y\right)\left\{F_{\left\{m_{r, c}\left(\alpha_{c}\right)\right\}}\right\} q^{n} \\
= & \sum_{n=0}^{\infty} \bigoplus_{\left\{m_{r}(c)\right\} \in \mathcal{P}_{n}\left(G_{*}\right)} \\
& \bigotimes_{r, c} \bigotimes_{\alpha_{c}=1}^{N_{c}} S^{m_{r, c}\left(\alpha_{c}\right)}\left(H\left(Y_{\alpha_{c}}^{c} / Z_{G}(c) ; x, y\right)\left\{F_{\alpha_{c}}^{c}+(r-1) d / 2\right\}\right) q^{n} \\
= & \sum_{\left\{m_{r}(c)\right\}} \bigotimes_{r, c} S^{m_{r, c}}\left(\bigoplus_{\alpha_{c}=1}^{N_{c}} H\left(Y_{\alpha_{c}}^{c} / Z_{G}(c) ; x, y\right)\left\{F_{\alpha_{c}}^{c}+(r-1) d / 2\right\} q^{r}\right) \\
= & \sum_{\left\{m_{r}\right\}} \bigotimes_{r} S^{m_{r}}\left(\bigoplus_{c}^{N_{c}} \bigoplus_{\alpha_{c}=1} H\left(Y_{\alpha_{c}}^{c} / Z_{G}(c) ; x, y\right)\left\{F_{\alpha_{c}}^{c}+(r-1) d / 2\right\} q^{r}\right) \\
= & \sum_{\left\{m_{r}\right\}} \bigotimes_{r \geq 1} S^{m_{r}}\left(H(Y, G ; x, y)\{(r-1) d / 2\} q^{r}\right) \\
= & S\left(\sum_{r \geq 1} H(Y, G ; x, y)\{(r-1) d / 2\} q^{r}\right) .
\end{aligned}
$$

Namely we have proved that

$$
\sum_{n \geq 0} H\left(Y^{n}, G_{n} ; x, y\right) q^{n}=S\left(\sum_{r>0} H(Y, G ; x, y)(x y)^{(r-1) d / 2} q^{r}\right),
$$

which implies immediately the theorem by means of Lemma 3.2 .

Remark 3.2. When $G$ is trivial and $Y$ is an algebraic surface, Theorem 3.1 recovers the orbifold Hodge numbers for the symmetric product $Y^{n} / S_{n}$ which was calculated in [16, 30]. On the other hand, if we set $x=y=1$ we recover the orbifold Euler numbers for $Y^{n} / G_{n}$ which was first computed in [27] for any topological space $Y$.

Remark 3.3. In the above we have constrained ourselves to the case that the shift numbers are integers. Physicists are also interested in the case of fractional shift numbers (see e.g. Zaslow [29]). It is straightforward to generalize our result.

3.3. Some consequences. We assume that $Y$ is a quasi-projective surface acted upon by a finite group $G$, and that $X$ is a resolution of singularities of the orbifold $Y / G$. We denote by $X^{[n]}$ the Hilbert scheme of $n$ points on $X$. It is well known (cf. 13, 15) that the Hilbert-Chow morphism $X^{[n]} \rightarrow X^{n} / S_{n}$ is a resolution of singularities. Indeed it is crepant. We have the following commutative diagram [27] 


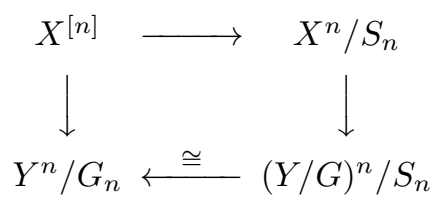

which implies that the Hilbert scheme $X^{[n]}$ is a resolution of singularities of the

orbifold $X^{n} / G_{n}$.

As calculated in [17] and [8], the Hodge numbers for the Hilbert scheme $X^{[n]}$ are given by the following formula:

$$
\sum_{n=1}^{\infty} e\left(X^{[n]} ; x, y\right) q^{n}=\prod_{r=1}^{\infty} \prod_{s, t} \frac{1}{\left(1-x^{s} y^{t} q^{r}(x y)^{r-1}\right)^{e^{s, t}(X)}} .
$$

Here and below we use the cohomology with compact supports.

By comparing with Theorem 3.1 we obtain the following theorem which provides us a large class of higher dimensional examples which verify the orbifold Hodge number conjecture (cf. [26, 29, 旬, 团). The assumption of the theorem is necessary by Remark 2.1.

Theorem 3.2. Let $Y$ be a smooth quasi-projective surface which admits a $G$-action with only isolated fixed points. Assume that $\pi: X \rightarrow Y / G$ is a resolution such that $e(X ; x, y)=e(Y, G ; x, y)$. Let $X^{[n]}$ be the Hilbert scheme of $n$ points of $X$. Then for all $r, s$ we have

$$
h^{r, s}\left(X^{[n]}\right)=h^{r, s}\left(Y^{n}, G_{n}\right) .
$$

Remark 3.4. When $G$ is trivial and $X$ equals $Y$, we recover the theorem of [16, 30]. We will see later many interesting examples arise when $G$ is not trivial.

More generally if $Y$ has dimension greater than 2, there is no such a favorable resolution as Hilbert scheme for $Y^{n} / G_{n}$. Nevertheless we have the following interesting corollary of Theorem 3.1. Here we assume that the shifts are integers for the orbifold $Y / G$ so that its orbifold Hodge numbers are well defined.

Corollary 3.1. Let $Y$ be a smooth variety of even dimension acted on by a finite group $G$ of automorphisms, and $\pi: X \rightarrow Y / G$ is a resolution such that $h^{s, t}(X)=$ $h^{s, t}(Y, G)$ for all $s, t$, then for all $s, t$ we have

$$
h^{s, t}\left(X^{n}, S_{n}\right)=h^{s, t}\left(Y^{n}, G_{n}\right) .
$$

\section{EXAMPLES AND APPLICATIONS}

In this section we provide various concrete examples which satisfy the assumptions of Theorem 3.1 and Theorem 3.2. We also give explicit conjectures on the elliptic genera for the wreath product orbifolds.

\subsection{Various examples.}

Example 4.1. When $G$ is trivial and $X$ equals $Y$, this gives us the example of symmetric products 15,30 . 
Example 4.2. $Y$ is $\mathbb{C}^{2}, G$ is a finite subgroup of $S L_{2}(\mathbb{C})$, and $X$ is the minimal resolution of $\mathbb{C}^{2} / G$. The exceptional fiber consists of $\left|G_{*}\right|-1$ irreducible components which are (-2)-curves (cf. e.g. [19]). We have

$$
h^{s, t}(X)= \begin{cases}1, & s=t=0 \\ \left|G_{*}\right|-1, & s=t=1 \\ 0, & \text { otherwise. }\end{cases}
$$

On the other hand, for any non-trivial conjugacy class $c \in G_{*}$, the corresponding shift is 1 and thus makes a contribution to $h^{1,1}\left(\mathbb{C}^{2}, G\right)$ which results that $h^{1,1}\left(\mathbb{C}^{2}, G\right)=\left|G_{*}\right|-1$. The other $h^{s, t}\left(\mathbb{C}^{2}, G\right)$ can be also seen to coincide with $h^{s, t}(X)$.

This example has played a key role in the connections between the wreath product orbifolds and the vertex representations of affine and toroidal Lie algebras 27, 14, 28.

Example 4.3. (Bryan-Donagi-Leung [7]) Let $Y$ be an abelian surface (two dimensional torus). The $\mathbb{Z}_{2}$-action induced by the involution $\tau: x \rightarrow-x$ has 16 fixed points, at each of which the shift $F^{\tau}$ is 1 . So the twisted sectors contribute an extra 16 to $h^{1,1}$. Write $Y=\mathbb{C}^{2} / L$ for some lattice $L$, and let $\left(z_{1}, z_{2}\right)$ be the linear coordinates on $\mathbb{C}^{2}$. Then $H^{*, *}(Y)$ is generated by $d z^{1}, d \bar{z}^{1}, d z^{2}, d \bar{z}^{2}$. The action of $\tau$ just takes $d z^{j}$ to $-d z^{j}$, etc. Hence it is clear that

$$
\begin{aligned}
H^{*, *}(Y)^{\mathbb{Z}_{2} \cong} & \mathbb{C} \oplus \mathbb{C} d z^{1} \wedge d z^{2} \oplus\left(\oplus_{j, k=1}^{2} \mathbb{C} d z^{j} \wedge d \bar{z}^{k}\right) \\
& \oplus \mathbb{C} d \bar{z}^{1} \wedge d \bar{z}^{2} \oplus \mathbb{C} d z^{1} \wedge d z^{2} \wedge d \bar{z}^{1} \wedge d \bar{z}^{2}
\end{aligned}
$$

Therefore,

$$
h^{s, t}\left(Y, \mathbb{Z}_{2}\right)= \begin{cases}1, & s=t=0 \\ 20, & s=t=1 \\ 1, & s=2, t=0 \text { or } s=0, t=2, \\ 1, & s=t=2, \\ 0, & \text { otherwise. }\end{cases}
$$

The minimal resolution $X \rightarrow Y / \pm 1$ is a crepant resolution, where $X$ is a K3 surface. This is the famous Kummer construction. By the well known Hodge numbers of a $\mathrm{K} 3$ surface, we have $h^{s, t}(X)=h^{s, t}\left(Y, \mathbb{Z}_{2}\right)$ for all $s, t$.

Example 4.4. Let $\mathbb{Z}_{3}$ act on $\mathbb{C P}_{2}$ by

$$
\alpha \cdot\left[z_{0}: z_{1}: z_{2}\right]=\left[\alpha z_{0}: \alpha^{-1} z_{1}: z_{2}\right],
$$

where $\alpha$ is a generator of $\mathbb{Z}_{3}$ and identified with a cubic root of unity on the righthand side. This action has three fixed points: $p_{0}=[1: 0: 0], p_{1}=[0: 1: 0]$, and $p_{2}=[0: 0: 1]$. At these point, the weights of the action are $(1,2),(2,1)$, and $(1,2)$ respectively. It is then straightforward to see that for $g \neq 1$ we have

Therefore,

$$
F^{g}=\frac{1}{3}+\frac{2}{3}=1
$$

$$
H^{*, *}\left(\mathbb{C P}_{2}, \mathbb{Z}_{3}\right)=H^{*, *}\left(\mathbb{C P}_{2}\right)^{\mathbb{Z}_{3}} \bigoplus \bigoplus_{j=0}^{2} H^{*, *}\left(p_{j}\right)^{\mathbb{Z}_{3}}\{1\} \bigoplus \bigoplus_{j=0}^{2} H^{*, *}\left(p_{j}\right)^{\mathbb{Z}_{3}}\{1\}
$$


and hence

$$
h^{s, t}\left(\mathbb{C P}_{2}, \mathbb{Z}_{3}\right)= \begin{cases}1, & s=t=0 \\ 7, & s=t=1 \\ 1, & s=t=2 \\ 0, & \text { otherwise }\end{cases}
$$

The minimal resolution $X=\widehat{\mathbb{C P}} / \mathbb{Z}_{3}$ is obtained by replacing each singular point by a string of two $(-2)$-curves, each of which contributes 1 to $h^{1,1}$, hence $h^{1,1}$ of $\widehat{C P}_{2} / \mathbb{Z}_{3}$ is 7 . This resolution is a crepant resolution.

Example 4.5. Let $n>2$ be an odd number, Consider the action of $\mathbb{Z}_{n}$ on $\mathbb{C P}_{3}$ given by

$$
\alpha \cdot\left[z_{0}: z_{1}: z_{2}: z_{3}\right]=\left[z_{0}: z_{1}: \alpha z_{2}: \alpha^{-1} z_{3}\right],
$$

where $\alpha$ is a generator of $\mathbb{Z}_{n}$. It has a fixed line $\left\{\left[z_{0}: z_{1}: 0: 0\right]\right\}$ and two isolated fixed points $[0: 0: 1: 0]$, and $[0: 0: 0: 1]$. Let $Y_{m, n}$ be the Fermat surface defined by

$$
z_{0}^{m n}+z_{1}^{m n}+z_{2}^{m n}+z_{3}^{m n}=0
$$

in $\mathbb{C P}_{3}$. The above action preserves $Y_{m, n}$, with $m n$ isolated fixed points:

$$
\left[1: e^{(2 k+1) \pi \sqrt{-1} /(m n)}: 0: 0\right], \quad k=0, \ldots, m n-1 .
$$

Note the action is semi-free, i.e. the stabilizers are either trivial or the whole group $\mathbb{Z}_{n}$. Near each of the fixed points, say $\left[1: e^{\pi \sqrt{-1} /(m n)}: 0: 0\right], Y_{m, n}$ is given by the equation

$$
1+u_{1}^{n}+u_{2}^{n}+u_{3}^{n}=0
$$

where $u_{j}=z_{j} / z_{0}$. We can use $\left(u_{2}, u_{3}\right)$ as local coordinates, then $\mathbb{Z}_{n}$ acts with weight $(1,-1)$, i.e., $\mathbb{Z}_{n}$ acts locally by matrices in $S L(2, \mathbb{C})$. Therefore, $Y_{m, n} / Z_{n}$ admits a crepant resolution obtained by replacing each isolated singular point with a string of $n-1$ copies of $(-2)$-curves.

Example 4.6. Denote now by $\beta$ a generator of $\mathbb{Z}_{4}$. Consider the $\mathbb{Z}_{4}$-action on $\mathbb{C P}_{3}$ given by

$$
\beta \cdot\left[z_{0}: z_{1}: z_{2}: z_{3}\right]=\left[z_{0}: z_{1}: \sqrt{-1} z_{3}: \sqrt{-1} z_{2}\right] .
$$

Combined with the $\mathbb{Z}_{n}$-action in Example 4.5, we get an action of the binary dihedral group $D_{n}^{*}$ on $\mathbb{C P}_{3}$ which preserves $Y_{4 m, n}$. By the same method as in Example 4.5 one can find the fixed points and sees that $Y_{4 m, n} / D_{n}^{*}$ admits a crepant resolution.

Example 4.7. The method of Example 4.5 and Example 4.6 can be generalized to other finite subgroups of $S L(2, \mathbb{C})$. Given such a group $G$, let it act on $\mathbb{C}^{4}$ on the last two factors. This action induces an action on $\mathbb{C P}_{3}$. Now consider a smooth hypersurface $Y$ defined by an equation of the form

$$
f\left(z_{0}, z_{1}\right)+g\left(z_{2}, z_{3}\right)=0,
$$

where $f$ and $g$ are two homogeneous polynomials of the same degrees, and $g$ is an invariant polynomial for $G$. Using the explicit description of the $G$-action on $\mathbb{C}^{2}$ and the invariant polynomials (see e.g. Klein [21]), one can find many examples which admits crepant resolutions. One should be able to find more examples by considering complete intersections in (weighted) projective spaces. 
Example 4.8. More complicated examples can be found in two papers by Barlow 2. 3] , e.g. the quotient of a Hilbert modular surface by $\mathbb{Z}_{2}$ or $D_{10}$, or the quotient of a complete intersection of 4 quadrics in $\mathbb{C P}_{6}$ by a group of order 16 , or the quotient of a Godeaux-Reid surface by an involution.

4.2. Conjectures on elliptic genera of wreath product orbifolds. Let $Y$ be a compact Kähler manifold of complex dimension $d$, denote by $T Y$ (resp. $T^{*} Y$ ) its holomorphic tangent (resp. cotangent) bundle. Consider the formal power series of vector bundles:

$$
E_{q, y}(Y)=y^{-\frac{d}{2}} \bigotimes_{n \geq 1}\left(\Lambda_{-y q^{n-1}}\left(T^{*} Y\right) \otimes \Lambda_{-y^{-1} q^{n}}(T Y) \otimes S_{q^{n}}\left(T^{*} Y\right) \otimes S_{q^{n}}(T Y)\right) .
$$

If we write

$$
E_{q, y}(Y)=\sum_{m \geq 0, l} q^{m} y^{l} E_{m, l}(Y)
$$

we easily see that each $E_{m, l}$ is a holomorphic bundle of finite rank, hence one can consider its Riemann-Roch number

$$
c(m, l)=\chi\left(E_{m, l}(Y)\right)=\sum_{k \geq 0}(-1)^{k} \operatorname{dim} H^{k}\left(Y, E_{m, l}(Y)\right) .
$$

The generating function

$$
\chi(Y ; q, y)=\sum_{m \geq 0, l} q^{m} y^{l} \chi\left(E_{m, l}(Y)\right)=\chi\left(E_{q, y}(Y)\right)
$$

is called the elliptic genus of $Y$ (cf. [18, 22] ). In the very important special case when $q=0$, one recovers the Hirzebruch genus:

$$
\begin{aligned}
& E_{0, y}(Y)=y^{-\frac{d}{2}} \Lambda_{-y}\left(T^{*} Y\right), \\
& \chi(Y ; 0, y)=y^{-\frac{d}{2}} \chi_{-y}(Y)=y^{-\frac{d}{2}} \sum_{s, t \geq 0}(-1)^{t}(-y)^{s} h^{s, t}(Y) .
\end{aligned}
$$

We do not know of a good mathematical formulation of elliptic genera for orbifolds. However physicists have interpreted elliptic genera as partition functions of supersymmetric sigma models, which makes sense also for orbifolds (cf. [22, 11 and references therein). Based on physical arguments and the description of fixedpoint sets for the symmetric group action on $Y^{n}$, Dijkgraaf et al 11] derived a formula for the elliptic genera of the symmetric products $S^{n}(Y)$ in terms of that of $Y$. In the case of a K3 surface or an abelian surface, they also conjectured that the same formula should compute the elliptic genera of the Hilbert schemes. Their method, if can be made mathematically rigorous, should also provide the proof of the following conjectures with suitable modifications. (In the following we denote by $\chi(Y, G ; q, y)$ the elliptic genera of an orbifold $Y / G$. )

Conjecture 4.1. Let $Y$ be a Kähler $G$-manifold. If we write the elliptic genus for $Y / G$ as $\chi(Y, G ; q, y)=\sum_{m \geq 0, l} c(m, l) q^{m} y^{l}$, then the elliptic genus for the wreath product orbifold $Y^{n} / G_{n}$ is given by the following formula:

$$
\sum_{N=0}^{\infty} p^{N} \chi\left(Y^{N}, G_{N} ; q, y\right)=\prod_{n>0, m \geq 0, l} \frac{1}{\left(1-p^{n} q^{m} y^{l}\right)^{c(n m, l)}} .
$$


WEIQIANG WANG AND JIAN ZHOU

Conjecture 4.2. Let $Y$ be a Kähler $G$-surface. We assume that $X$ is a resolution of singularities of $Y / G$ such that $\chi(Y, G ; q, y)=\chi(X ; q, y)$. Then $\chi\left(Y^{n}, G_{n} ; q, y\right)=$ $\chi\left(X^{[n]} ; q, y\right)$ for all $n$.

When $G$ is trivial, one recovers the symmetric product situation as in [11]. In this case, the $q=0$ version of Conjecture 4.1 has been verified in [30] as a corollary of the calculation of orbifold Hodge numbers. Similarly, our results in Section 3 can be viewed as supporting evidence for the above conjectures in the general setup of wreath product orbifolds.

Note added. In a recent remarkable paper [6], Borisov and Libgober have introduced the mathematically rigorous notion of orbifold elliptic genera among other things, and verified our Conjecture 4.1 .

\section{REFERENCES}

[1] M. Atiyah, G. Segal, On equivariant Euler characteristics, J. Geom. Phys. 6 (1989), no. 4, 671-677.

[2] R. Barlow, Some new surfaces with $p_{g}=0$, Duke Math. J. 51 (1984), no. 4, 889-904.

[3] R. Barlow, A simply connected surface of general type with $p_{g}=0$, Invent. Math. 79 (1985), no. 2, 293-301.

[4] V.V. Batyrev, Stringy Hodge numbers of varieties with Gorenstein canonical singularities. In: Integrable systems and algebraic geometry (Kobe/Kyoto, 1997), 1-32, World Sci. Publishing, River Edge, NJ, 1998.

[5] V.V. Batyrev, D. Dais, Strong McKay correspondence, string-theoretic Hodge numbers and mirror symmetry, Topology 35 (1996), no. 4, 901-929.

[6] L. Borisov and A. Libgober, Elliptic genera of singular varieties, preprint, math.AG/0007108.

[7] J. Bryan, R. Donagi, and N.C. Leung, G-bundles on Abelian surfaces, hyperKähler manifolds, and stringy Hodge numbers, preprint, math.AG/0004159.

[8] J. Cheah, On the cohomology of Hilbert schemes of points, J. Alg. Geom. 5 (1996), no. 3, 479-511.

[9] P. Deligne, Théorie de Hodge. II., Inst. Hautes Études Sci. Publ. Math. 40 (1971), 5-57.

[10] J. Denef and F. Loeser Motivic integration, quotient singularities and the McKay correspondence, preprint, math.AG/9903187.

[11] R. Dijkgraaf, G. Moore, E. Verlinde, and H. Verlinde, Elliptic genera of symmetric products and second quantized strings, Commun. Math. Phys. 185 (1997) 197-209.

[12] L. Dixon, J.A. Harvey, C. Vafa, E. Witten, Strings on orbifolds, Nuclear Phys. B 261 (1985), no. $4,678-686$

[13] J. Fogarty, Algebraic families on an algebraic surface, Amer. J. Math. 90 (1968), 511-521.

[14] I. B. Frenkel, N. Jing and W. Wang Vertex representations via finite groups and the McKay correspondence, (math.QA/9907166), Internat. Math. Res. Notices, No. 4 (2000) 195-222.

[15] L. Göttsche, The Betti numbers of the Hilbert scheme of points on a smooth projective surface, Math. Ann. 286 (1990) 193-207.

[16] L. Göttsche, Orbifold-Hodge numbers of Hilbert schemes. In: Parameter spaces (Warsaw, 1994) 83-87. Polish Acad. Sci., Warsaw, 1996.

[17] H. Göttsche and W. Soergel, Perverse sheaves and the cohomology of Hilbert schemes of smooth algebraic surfaces, Math. Ann. 296 (1993) 235-245.

[18] F. Hirzebruch, Elliptic genera of level $N$ for complex manifolds. In: Differential geometrical methods in theoretical physics (Como, 1987), 37-63, NATO Adv. Sci. Inst. Ser. C: Math. Phys. Sci., 250, Kluwer Acad. Publ., Dordrecht, 1988.

[19] F. Hirzebruch and T. Höfer, On the Euler number of an orbifold, Math. Ann. 286 (1990) $255-260$.

[20] A. Kerber, Representations of permutation groups. I. Lect. Notes in Math. 240. SpringerVerlag, Berlin-New York, 1971.

[21] F. Klein, Lectures on the icosahedron and the solution of equations of the fifth degree. Translated by G.G. Morrice, second and revised edition, London, 1913.

[22] P.S. Landweber (Ed.), Elliptic curves and modular forms in algebraic topology, lect. Notes in math. 1326. Springer-Verlag, 1988. 
[23] I. Macdonald, Symmetric Functions and Hall Polynomials, Second Edition, Oxford, Clarendon Press, 1995.

[24] M. Reid, La correspondance de McKay, Séminaire Bourbaki, 52ème année, novembre 1999, no. 867, to appear in Astérisque 2000.

[25] I. Satake, On a generalization of the notion of manifold, Proc. Nat. Acad. Sci. U.S.A. 42 (1956), 359-363.

[26] C. Vafa, String vacua and orbifoldized LG models, Modern Phys. Lett. A4 (1989) 1169-1185.

[27] W. Wang, Equivariant K-theory and wreath products, MPI preprint \# 86, August 1998; Equivariant K-theory, wreath products and Heisenberg algebra, (math.QA/9907166), Duke Math. J. 103 (2000) 1-23.

[28] W. Wang, Hilbert schemes, wreath products, and the McKay correspondence, preprint, math.AG/9912104.

[29] E. Zaslow, Topological orbifold models and quantum cohomology rings, Commun. Math. Phys. 156 (1993), no. 2, 301-331.

[30] J. Zhou, Delocalized equivariant cohomology of symmetric products, math.DG/9910028, preprint.

Department of Mathematics, North Carolina State University, Raleigh, NC 27695

E-mail address: wqwang@math.ncsu.edu

Department of Mathematics, Texas A\&M University, College Station, TX 77843

E-mail address: zhou@math.tamu.edu 